\title{
La incidencia de los eventos científico técnicos en los activos intangibles de sus actores. Caso de estudio: pedagogía 2019
}

The incidence of technical scientific events on the intangible assets of its actors. Case study: pedagogy 2019

Lic. Ailim Sánchez Fernández. ${ }^{1}$

\begin{abstract}
The events as communicative pieces respond fully to the Knowledge Society, and their effectiveness for the acquisition of this is specified to a large degree in those with scientific-technical themes. However, if this benefit is completely explicit for the participants, texts or publications of this impact do not appear in the same way in the organizing actors of the same. In Cuba, specifically, business and public, tourist and nontourist entities are integrated in the organization and execution of scientific and technical events, so that the economic benefits, tangible although they constitute an economic requirement of the country, are not unique, and the intangibles They point to a strategic vision. This situation promotes the general objective of the research defined as Identifying the benefits provided by technical scientific events to the development of intangible assets of its actors and the destination of Havana, whose fulfillment supports its application in the XVI Edition of the International Congress of Pedagogy as case study.

To fulfill this objective, theoretical methods such as bibliographic and documentary analysis and empirical methods were used, such as the application of surveys to eventists and conducting interviews with specialists from the different actors in the management of events. The results achieved form a guideline to be followed for the evaluation of other congresses with a similar nature and provide visibility to the importance of events of this nature, by determining that they contribute to the development of the human, structural and relational capital of their actors.
\end{abstract}

Keywords: Knowledge, Intangibles, Scientific-Technical Events, International Pedagogy Congress, Actors Of The Event

\section{Resumen}

${ }^{1}$ Universidad de La Habana, Facultad de Turismo, ailim931204@gmail.com 
Los eventos como piezas comunicativas responden plenamente a la Sociedad de Conocimiento, y su efectividad para la adquisición de este se concreta en amplio grado en aquellos con temáticas científico técnicas. Sin embargo, si este beneficio es totalmente explicito para los participantes, no aparecen de igual forma textos o publicaciones de este impacto en los actores organizadores de los mismos. En Cuba, específicamente, se integran en la organización y ejecución de eventos científico técnico, entidades empresariales y públicas, turísticas y no turísticas, por lo que los beneficios económicos, tangibles aunque constituyan una exigencia económica del país, no son únicos, y los intangibles apuntan a una visión estratégica. Esta situación promueve el objetivo general de la investigación definido como Identificar los beneficios aportados por los eventos científico técnicos al desarrollo de los activos intangibles de sus actores y el destino La Habana, cuyo cumplimiento sustenta su aplicación en la XVI Edición del Congreso Internacional de Pedagogía como estudio de caso.

Para el cumplimiento de este objetivo se emplearon métodos teóricos como el análisis bibliográfico y documental y empíricos como la aplicación de encuestas a eventistas y la realización de entrevistas a especialistas de los diferentes actores en la gestión de los eventos. Los resultados alcanzados conforman una pauta a seguir para la valoración de otros congresos con naturaleza similar y brindan visibilidad a la importancia de los eventos de esta naturaleza, al determinarse que contribuyen al desarrollo del capital humano, estructural y relacional de sus actores.

Palabras Clave: Conocimiento, Intangibles, Eventos Científico Técnicos, Congreso Internacional Pedagogía, Actores del Evento

\section{Introducción}

La realización de eventos, satisface en primera instancia una necesidad comunicativa y adopta en función de esta, dinámicas diferentes. El comportamiento de estos encuentros humanos, hoy en parte digitalizados, mostró un amplio desarrollo en el Siglo XX, incidido por los procesos tecnológicos, económicos y sociales, que se produjeron en ese marco temporal y continúan en la actualidad, a tal punto de definir un nuevo profesional: Organizador Profesional de Congresos/Eventos (OPC/OPE) y una modalidad turística, de hecho vinculados desde la génesis al nacimiento del turismo como negocio: Thomas Cook, el viaje organizado y la reunión de alcohólicos anónimos. De tal forma, que siendo los eventos motivación de viaje, se conforma como un negocio para empresas, destinos turísticos y un conjunto de instituciones vinculadas a la actividad que conforman una cadena productiva sui generis.

Múltiples autores han abordado los impactos positivos del Turismo de Eventos, sin embargo, las investigaciones son aún insuficientes de forma general, así como en el entorno cubano. Si bien, por una parte, el extinto Buró de Convenciones de Cuba, diseñó el Sistema de Estadísticas Complementario para Eventos e Incentivos, desactivado después de la desaparición de este órgano, la evaluación del impacto de los eventos, desde el punto de vista cualitativo, continúa siendo una asignatura pendiente en el país. 
De hecho los eventos, los flujos turísticos que generan y por tanto los ingresos han sido catalogados como Turismo Alternativo, Turismo Profesional o Turismo Especializado, soslayando los enfoques convencionales del turismo y de alguna forma aproximándose a la sostenibilidad. No obstante, esta queda limitada a elementos cuantitativos y económicos aun cuando en su propia definición comprende aquellas actividades turísticas respetuosas con el medio natural, cultural y social, y con los valores de una comunidad, que permiten disfrutar de un positivo intercambio de experiencias entre residentes y visitantes, donde la relación entre el turista y la comunidad es justa, y donde los visitantes tienen una actitud verdaderamente participativa en su experiencia de viaje (http://www.turismo-sostenible.org). Gandara (2001), coherente con los elementos anteriores introduce en la definición de sostenibilidad de los destinos turísticos, el adecuado desarrollo y la acertada gestión de un destino.

Varios autores, especialmente para los eventos, entre los que se encuentra Eulogio Rodríguez (2001), han destacado la multiplicidad de aristas de los impactos positivos de los flujos que ellos motivan, al plantear que "la contribución del turismo de eventos al desarrollo turístico de cualquier país es incuestionable. No solo por el volumen de ingresos y de turistas se resume su significativa contribución, sino también como promotor del turismo y conformador de una nueva imagen, acerca de un destino, es ahí quizás donde radica la mayor importancia estratégica que tiene y tendrá esta modalidad para presentes y futuras generaciones", mientras que Getz (2008) a su vez valora a los eventos como un importante motivador de turismo, una figura prominente en los planes de desarrollo y marketing, y apunta a la importancia creciente de los mismos para la competitividad del destino. Este último postulado remite a la conceptualización de los destinos turísticos y la estrecha relación que existe entre la competitividad de los mismos y la de los actores residentes, por lo que más allá de la sostenibilidad del evento en sí, determinada en cierta medida desde el año 2012 a partir de la Norma ISO 20121, es necesario estudiar el cómo inciden los eventos en la competitividad de los actores y del destino mismo, cómo contribuyen a crear y fortalecer fundamentos de ventajas competitivas en un entorno determinado, bajo la consideración de la paridad competitividad - sostenibilidad. "El turismo es resultado de las condiciones económicas, políticas y sociales de una sociedad en un momento específico, por ello depende del estilo de desarrollo en el que se inserte. Para un desarrollo factible del turismo sostenible, la cuestión es la identificación de las necesidades sociales.” (Bertoni, 2008).

En la actualidad, y definido hace más de dos décadas por Drucker (1993) en el contexto nación, la clave para la construcción de la riqueza es el conocimiento, de tal forma que se relaciona el conocimiento como fuente de ventajas competitivas de las instituciones, si bien la literatura actual se enfoca en gran parte a las organizaciones empresariales. Una revisión bibliográfica realizada por un colectivo de autores, resume que "Actualmente se acepta que, en las sociedades económicamente desarrolladas y tecnológicamente avanzadas, el conocimiento constituye un pilar básico en la creación de ventaja competitiva... En este nuevo escenario socioeconómico así configurado, la capacidad de generación de riqueza de las empresas no se entiende ya relacionada con la eficiencia en 
la gestión de los activos materiales; muy al contrario. La generación y el mantenimiento de ventajas competitivas, que determinan la capacidad de creación de valor para los propietarios, están estrechamente vinculados a la realización de inversiones intangibles y a la eficiencia con la que se gestionan los denominados activos intangibles". (Garcia, Simo, \& Joan Mundet, 2004)

Los eventos por su parte como piezas comunicativas responden plenamente a esta Sociedad de Conocimiento, y su efectividad para la adquisición de este se concreta en amplio grado en aquellos con temáticas científico técnicas. Sin embargo, si este beneficio es totalmente explicito para los participantes, no aparecen de igual forma textos o publicaciones de este impacto en los actores organizadores de los mismos. En Cuba, específicamente, se integran en la organización y ejecución de eventos científico técnico, entidades empresariales y públicas, turísticas y no turísticas, por lo que los beneficios económicos, tangibles aunque constituyan una exigencia económica del país, no son únicos, y los intangibles apuntan a una visión estratégica.

Se define así el objetivo general de la investigación Identificar los beneficios aportados por los eventos científico técnicos al desarrollo de los activos intangibles de sus actores y el destino La Habana.

Para el cumplimiento del mismo, se tomó como caso de estudio el Congreso Internacional Pedagogía 2019, a partir de su carácter de evento de diseño propio (generado por una institución nacional), su madurez (dieciséis ediciones), su tamaño (megaevento) y la coherencia del tema con la imagen orgánica país.

La investigación constituye, según conocimiento de la autora, el primer ejercicio para una evaluación de esta naturaleza de un evento, toda vez, que aun con las interacciones y coordinaciones existentes entre la sede, la agencia y el comité organizador, construidas en las múltiples ediciones del evento en estudio, los intereses con relación al mismo son diferentes, y en función de los mismos se valora en cada institución. De tal forma, que la metodología empleada puede ser punto de partida para análisis similares en eventos de interés.

\section{Desarrollo}

Para el cumplimiento del objetivo anteriormente expuesto se definió un procedimiento metodológico, el cual, de conjunto con sus contenidos se detalla a continuación:

1) Caracterización del Evento: Diseñado en 1986 por el Ministerio de Educación (MINED) a partir de la idea y liderazgo del Comandante en Jefe Fidel Castro Ruz, para que educadores de Cuba y el mundo intercambien experiencias, dialoguen y polemicen, basados en el respeto a la diversidad de opiniones, construyan un mundo mejor, el Congreso Internacional Pedagogía es uno de los eventos internacionales más antiguos y prestigiosos de la cartera cubana, el de mayor participación que ha contado en total con la presencia de más de 78000 educadores de América Latina y otras partes del mundo. El Congreso se convierte en una expresión y vía del cumplimiento de la misión del MINED de dirigir, 
ejecutar y controlar la aplicación de la política del Estado y el Gobierno en la actividad educacional, excepto en la Educación Superior; para lo cual ha dirigido sus esfuerzos al perfeccionamiento del sistema nacional, a la consolidación de la formación y superación del claustro de profesores y a darle integralidad al sector, en armonía con los requerimientos del desarrollo económico y social del país, convirtiendo los centros educacionales en instituciones culturales de la comunidad. Tanto por el volumen, como por el número de ediciones celebradas, el Congreso Internacional Pedagogía constituye un evento de diseño propio exitoso, que justifica su elección como caso a estudiar en los eventos científicos técnicos del país. El evento está íntimamente vinculado con la imagen país (Educación) y se clasifica como "grande" solo con la participación extranjera (más de 1000 participantes) y como "mega-evento" con participación total (incluyendo nacionales).

El Congreso Internacional Pedagogía 2019, celebrado del 4 y al 8 de febrero, en su XVI edición y bajo el lema: "Por la unidad de los educadores", socializó resultados científicos y buenas prácticas del quehacer de miles de profesionales de la educación que dan respuesta a los apremiantes problemas relativos a la Educación para lograr una educación de calidad desde una dimensión integradora, dirigida a contribuir decisivamente en el cumplimiento de los Objetivos de Desarrollo Sostenible de la Agenda 2030. Contó con la presencia de 44 países de los cuales 1308 fueron delegados, 9 delegados no presenciales, 12 expositores y 21 invitados, para un total de acreditados de 1350.

2) Identificación de los actores del Evento: Palacio de las Convenciones (sede), MINED (Comité Organizador), Cubatur (Agencia de Viajes receptiva) y Delegación de la Habana, Mayabeque y Artemisa (Gestor de destino).

3) Definición de los conceptos básicos

Beneficios intangibles: Son aquellos que se obtienen por la mejora del capital humano, estructural y relacional de los actores fundamentales del evento, o sea que favorece la creación y flujo del conocimiento, la capacidad de innovación, los canales de distribución, la accesibilidad al mercado, la imagen de marca, la lealtad y captación de clientes y las alianzas estratégicas. (Pujol \& Maroto, 2003), (Villa, 2014).

Las economías nacionales han sufrido una transformación y han dejado de guiarse por criterios propios de la era industrial para apoyarse en los nuevos modelos de la era del conocimiento, donde el capital intelectual juega un papel decisivo en el desarrollo de los países. Por ejemplo, Holanda fue pionera en la inclusión en las cuentas nacionales de las inversiones en activos intangibles y las oficinas de estadística de países como Italia o Suecia efectúan estudios periódicos sobre las inversiones en intangibles a nivel macroeconómico. Malgor (2013)

El capital intelectual, entendido como el conjunto de activos de un territorio que, careciendo de naturaleza física o financiera, generan o pueden generar desarrollo sostenible, solos o en conjunción con otros, presenta seis dimensiones: capital turismo, capital actividad económica, capital social, capital medioambiental, capital 
administración pública y capital formación y desarrollo. Cada una de estas seis dimensiones integra elementos intangibles, estratégicos para el crecimiento, el progreso y el bienestar de los habitantes de cada espacio a promover: la calidad de sus alojamientos turísticos, la productividad y competitividad de sus empresas, el empleo, la vivienda, la cultura, la calidad del aire, la conservación del medio ambiente y de los recursos naturales, la eficiencia de sus instituciones públicas, la educación, la implantación de nuevas tecnologías, etc.

Según Malgor (2013), todo territorio, para ser competitivo, debería estudiar y administrar adecuadamente su capital intelectual o intangible, ya que en la actualidad se sabe que tiene más importancia la manera en que se gestionan los recursos que los propios recursos, y que de esa gestión se obtiene una mejor comprensión de sus elementos diferenciales, lo que a su vez genera mayor bienestar social y ventajas competitivas respecto a otras regiones.

Según Santander Convention Bureau, cuya ciudad en 2018 presento cifras positivas como destino MICE, para seguir con esta línea de crecimiento, "los profesionales de esta industria creen necesario una mejora de las comunicaciones con la región". Para el 2019, dentro de su estrategia se planificó mantener la progresión llevando a cabo una fuerte apuesta por la cultura y la singularidad de contar en un radio de 500 metros con importantes atractivos turísticos.

El informe del Spain Convention Bureau (SCB) ofrece un análisis cualitativo de la situación del sector, que parte de la opinión de los 57 destinos que lo constituyen. Entre los principales puntos fuertes identificados en Revista Hosteltur (2020) se encuentran:

1. Ofertas existentes en turismo de reuniones, tanto de infraestructuras específicas como de oferta complementaria.

2. Posicionamiento del sector, percepción del destino por parte del mercado internacional.

3. La amplitud de la oferta dado por la variedad de destinos que se pueden ajustar a las diferentes demandas, con conectividad entre ellos, con percepción de seguridad y atractivo, con buena relación calidad/precio, con infraestructuras renovadas y variedad de actividades complementarias.

4. Para progresar en este ámbito, el sector debe adaptarse a la demanda, actualizarse y responder a las necesidades del cliente de forma rápida. Cada vez se buscan más experiencias nuevas e impactantes

5. Potenciar los destinos de tamaño medio y bajo

6. Mejorar en la captación de reuniones internacionales. Entre los factores en los que se detecta capacidad de mejora, juega también un papel fundamental una mayor promoción, algo que demandan todos los destinos.

De manera general, se define como beneficios intangibles a aquellos que se obtienen por la mejora del capital humano, estructural y relacional de los actores fundamentales del evento, o sea que favorece la creación y flujo del conocimiento, la capacidad de innovación, los canales de distribución, la accesibilidad al mercado, la imagen de marca, 
la lealtad y captación de clientes y las alianzas estratégicas (Pujol \& Maroto, 2003), (Villa, 2014). Dónde:

- El Capital Humano, es la fuerza que hay detrás del intelecto humano y la innovación de una empresa (Martínez, 2003), el conocimiento que poseen las personas, su capacidad de aprender, experiencias, creatividad, su actitud (motivación de los empleados y capacidad de liderazgo de la alta dirección) y demás características que hacen parte del talento humano en la organización. (Villa, 2014)

- El Capital Estructural es el conjunto de activos intangibles capaces de generar conocimiento, ubicados en el ámbito de acción de la organización. Este conocimiento se presenta en forma explícita, codificada y sistematizada, a la vez que es interiorizado por la Organización mediante un proceso formal que opera a través de la creación de una sucesión de rutinas organizativas o de pautas de acción que van siendo sistematizadas y socializadas por la Organización (Centro de Investigación sobre la Sociedad del Conocimiento, 2003, citado por Villa, 2014).

- El Capital Relacional surge de la interacción de la empresa y el mercado, se define como la habilidad de la empresa para interactuar de forma positiva con los miembros de la comunidad de negocio para estimular el potencial para la creación de riqueza, mejorando el capital humano y estructural (Villa, 2014). Incluye clientes, proveedores y socios.

4) Determinación de indicadores a valorar.

- Adquisición de conocimientos y habilidades

- Fomento de la innovación en la organización

- Posibilidad de intercambio

- Ampliación y /o fortalecimiento de los canales de distribución de sus productos y servicios

- Creación y/o fortalecimiento de nuevos grupos o redes para la mejora de su trabajo

- Acceso a nuevos grupos de clientes

- Fortalecimiento la lealtad de sus clientes

- Fortalecimiento su imagen de marca

- Captación de Ingresos Directos (aquellos vinculados a la actividad fundamental e inmediata)

- Captación de Ingresos Indirectos (aquellos derivados de la actividad fundamental para la actualidad y el futuro)

- Fortalecimiento de su capital humano

- Mejora de su capacidad asociativa e integradora

- Posibilidad de alianzas estratégicas

5) Diseño de las herramientas (Entrevista y cuestionarios): Fueron entrevistados especialistas de los actores identificados, de forma semiestructurada, solicitando sus criterios sobre en qué medida el Congreso Pedagogía facilitaba los indicadores definidos 
anteriormente, lo que posibilitó su estandarización, la profundización y la mayor información de cada criterio. A los efectos de la cuantificación de las respuestas cualitativas, cada criterio se evalúa de Muy Alto (5); Alto (4); Medio (3); Bajo (2) y Muy Bajo (1).

Se incorporó dentro de los actores Solways dado que los resultados de una investigación cuantitativa paralela arrojaron que fue el canal de distribución más utilizado por los participantes.

Se aplicó un cuestionario a eventistas para caracterizar su comportamiento como eventista-turista y su intención de compra. (Anexo 1)

6) Determinación de la muestra: Se utilizó un muestreo simple probabilístico, a partir del número de participantes extranjeros, calculado por tabla Excel disponible en http://www.plantillasmil.com. (Anexo 2). Los resultados fueron procesados con el Sistema SPSS Statistics 20.

7) Aplicación de las herramientas: Los cuestionarios fueron aplicados en el marco temporal del Congreso, y las entrevistas realizadas en los centros de los actores, posterior al mismo, con el objetivo de disponer de mayor tiempo para el intercambio. Fueron entrevistados cinco especialistas de los actores identificados, así como una especialista de la Agencia de Viajes HAVANATUR como agencia receptiva líder de eventos, con el fin de conformar la visión "destino" y ampliar las agencias de viajes por su rol jugado como distribuidor de la edición del 2019 y la experiencia de sus miembros en la gestión de eventos.

8) Valoración de la información: Es importante señalar que la naturaleza de las instituciones entrevistadas, el rol que cada una juega en la gestión del Congreso Internacional Pedagogía 2019 y las incidencias o impactos de su ejecución incidió en sus contestaciones, lo que se aprecia a partir de la desviación standard de la cuantificación de las mismas. (Anexo 3)

Mientras que los entrevistados de las Agencias de Viajes, la Delegación y el MINED consideran como alta o muy alta, la adquisición de conocimientos, Palacio de las Convenciones la valora como media al considerar que la madurez del evento, su carácter de organizador profesional, y lo que ello implica, la celebración del evento conforma un espacio para adquirir conocimientos organizacionales solo de forma moderada, a pesar de que cada edición es un ejercicio único. Así mismo su componente innovador puede limitarse, toda vez que su diseño se estandariza, por lo que el aporte innovador del evento no depende de este en sí, sino en el vínculo establecido con el Comité Organizador (MINED) y la profesionalidad del OPC, aunque de hecho la propia gestión de eventos es altamente innovadora.

Desde la visión de Solways, todos los eventos tienen sus particularidades y de todos se aprende algo ya sea mediante la gestión de los diferentes servicios que solicita el Comité Organizador como la confección de paquetes turísticos especializados que deben ofrecer las agencias de viajes. Se fomenta así tanto la innovación en el evento en sí, como en las 
organizaciones implicadas. La propia decisión en esta edición de Pedagogía de los ponentes no presenciales constituyó una innovación. La creación de nuevas alternativas antes las posibles dificultades que puede tener un evento solo se logran si se trabaja de conjunto con todas las organizaciones involucradas, para lo cual es necesario un flujo de información constante.

Debido a la complejidad y magnitud de este tipo de evento se crean nuevas actividades según el Programa Científico del mismo, en ocasiones se requiere una alta operación de transporte para reuniones especiales con ministros y personalidades, exigiendo nueva dinámica y logística que favorecen el surgimiento y desarrollo de habilidades de los especialistas de la agencia de viajes. Por otra parte, como receptivo, la improvisación es el factor clave del éxito a través de una toma de decisiones en tiempo.

Ejemplo de innovación de esta edición constituyó la impartición de conferencias magistrales por personalidades de la cultura y la historia y el otorgamiento por vez primera de reconocimientos a los Mejores Maestros Investigadores del país.

El Comité Organizador (MINED) considera que las presentaciones en directo de los estudios y avances que exponen los propios investigadores ayudan a una mejor comprensión del tema que en ocasiones derivan en un gran debate. Además, es un factor fundamental para la mejora continua de cada edición del Congreso, a partir de los diferentes encuentros académicos que se realizan durante la semana.

De tal forma el propio evento ayuda a la creación de nuevos mecanismos para ofrecer un Programa Científico lo más completo posible ya sea a través de las visitas a instituciones, actividades especiales, programas como por ejemplo "Yo sí puedo". Aun cuando todos los participantes persiguen un objetivo común "la educación", cada edición es diferente y las temáticas varían según las tendencias actuales y los intereses de cada país.

Sin embargo, las agencias de viajes entrevistadas consideran que las temáticas deberían ser renovadas.

La Delegación, por su parte, reconoce la importancia del evento, pero el evento no constituye en sí un elemento innovador, toda vez que forma parte de la oferta esperada del destino y la propia Delegación no se inserta en sus procesos de gestión. Esta misma posición fundamenta que la Delegación sea la institución entrevistada que menos realce la posibilidad de intercambio. No obstante, alega que aún con la visión del evento como un producto académico, el poder adquisitivo medio - bajo de sus participantes, debe ser considerado para la creación de nuevas ofertas turísticas, que posibiliten, a partir de la interacción de todos los actores una fusión, para que el producto global sea satisfactorio, motivador y atractivo. Consideran, además, que en la actualidad no se aprovecha al máximo al eventista, que se deben crear estrategias de promoción y de venta que atraigan al cliente a la compra de paquetes turísticos. Los eventos son grandes generadores de ingresos si se planifican bien las actividades y se diseñan ofertas personalizadas de acuerdo con las características del eventista y de los hoteles. 
Todos los entrevistados valoran de alta la posibilidad que brinda el evento en cuanto la ampliación o fortalecimiento de los canales de distribución, aunque, no se valora en igual forma su rol como vía para la atracción de nuevos grupos de clientes. En contraposición con ello, el cuestionario arroja que el $15 \%$ de los encuestados no había visitado la Ciudad y está en disposición de regresar al próximo Congreso Internacional Pedagogía 2021, a cualquier otro congreso, curso o de vacaciones.

La creación o el fortalecimiento de grupos o redes para la mejora del trabajo es reconocida por todos los encuestados. Por su parte el Comité Organizador plantea que el intercambio de experiencias durante el congreso crea nuevos vínculos entre instituciones y profesionales lo cual implica el enriquecimiento social, cultural y personal. Se crean las redes, pero no hay sistematicidad del trabajo en las diferentes temáticas.

Ninguno de los entrevistados valora altamente el evento como forma para fortalecer en mayor grado la lealtad de sus clientes, cuando el $24 \%$ de los encuestados ya han participado en otros Congresos Internacionales de Pedagogía y de ellos el $71 \%$ piensa volver a otra edición. El evento constituye así un espacio en el cual todos los actores pudieran realizar acciones de monitoreo y fidelización.

De la entrevista con Solways se extrae que "Cada evento es un nuevo grupo de clientes que se inserta en la agencia y que se le debe dar un seguimiento a sus participantes ya sea para asistir a un congreso o venir a vacacionar. Si el participante queda satisfecho con la atención brindada durante toda su estancia a través de los diferentes servicios que ofrece la Agencia, existe una gran posibilidad de que repita y que lo recomiende a sus compañeros."

Los actores directos (Palacio de las Convenciones y MINED) y Solways consideran el evento como muy importante para el fortalecimiento de su imagen de marca.

Casi la totalidad de los encuestados $(99,1 \%)$ recomenda el evento y según la encuesta realizada por el Comité Organizador el 95\% de los encuestados manifestaron deseo de participar en el próximo Congreso de Pedagogía, tributando así a la imagen de marca del Palacio de Convenciones y el MINED. A pesar de la nominación de La Habana como "Ciudad de Encuentros", la Delegación no percibe en toda su magnitud, el nivel de notoriedad potencial que representa que el $98 \%$ de los encuestados recomienden una visita a la Ciudad.

Esta visión divergente entre los principales actores y la delegación no posibilitó la promoción en el evento, considerado uno de los mayores del país, del 500 Aniversario de la Capital.

Todos los entrevistados valoran en alto - muy alto grado el evento como vía de fortalecimiento de su capital humano y mejora de su capacidad asociativa e integradora, así como que brinda la posibilidad para el establecimiento de alianzas estratégicas.

Desde su rol tanto de organizadora como de receptiva, en la etapa de ejecución de un evento, la atención personalizada al participante y el control y seguimiento a las actividades programadas ayudan a los especialistas de la agencia a tener un mayor 
dominio y experiencia de su trabajo, así como a aumentar el poder de toma de decisiones ante cualquier situación.

Dada la naturaleza del trabajo pedagógico, o sea, la necesidad de investigación, información y actualización del personal vinculado al mismo, el evento constituye un espacio u oportunidad muy valorada por el MINED para el desarrollo de su capital humano, por lo que se intenta que todas las Universidades de Ciencias Pedagógicas del país participen y que roten a los estudiantes seleccionados. La participación cubana en el evento fortalece la propia imagen del mismo, y la imagen orgánica del MINED y del país, reforzada por la presencia de organismos y personalidades internacionales.

El rol de promotor del turismo de esta modalidad se valida con los resultados del cuestionario que muestra, a partir de la interrelación existente entre la primera vez en La Habana y la primera vez al Congreso, que más del $65 \%$ de los encuestados acude al destino por el evento. No obstante, no se aprovechan al máximo las oportunidades que brindan las tecnologías actuales de la información y las comunicaciones, el seguimiento y actualización del sitio web del evento es insuficiente, carece de presencia en las redes sociales como Facebook, Instagram y Twitter; aspecto además de importancia para el incremento del número de delegados, cuando los resultados del cuestionario realizado en esta edición muestran que la vía por la que tuvieron información sobre el congreso fue Internet, básicamente por el sitio Web del Congreso y Facebook, aun cuando el propio evento no está como tal en esta red.

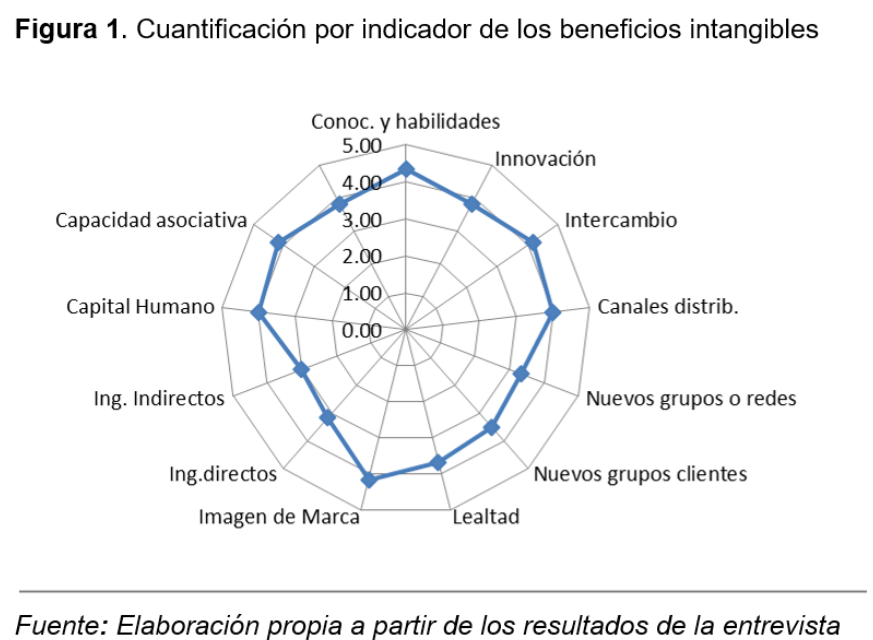

Fuente: Elaboración propia a partir de los resultados de la entrevista

Los indicadores seleccionados para valorar los intangibles, muestran que:

- Las mayores evaluaciones corresponden a la Adquisición de conocimientos y habilidades y Posibilidad de Intercambio, dado por las características de un Congreso y de los eventos en general respectivamente, mientras que los procesos de gestión y complejidad del evento favorecen la Capacidad asociativa o integradora. 
- Las peores evaluaciones corresponden a los indicadores vinculados con los ingresos, sustentados en la tendencia decreciente del número de participantes extranjeros y la débil comercialización del paquete turístico.

- El Congreso representa una oportunidad no totalmente aprovechada para el desarrollo de otros indicadores como el fortalecimiento de la lealtad de los clientes y la creación y fortalecimiento de grupos o redes.

- No se visualiza óptimamente el evento como espacio para acceder a nuevos grupos de clientes.

\section{Conclusiones}

- El análisis de los intangibles arroja que el Congreso Internacional Pedagogía contribuye en grado medio alto al desarrollo del Capital humano, estructural y relacional de sus actores.

- Es importante relacionar los beneficios con el desarrollo de las competencias organizacionales, y por tanto del Capital Intelectual del MINED, del Grupo Empresarial Palco y las Agencias de Viaje que posibilitaría a mayor plazo un mejor diseño de producto, una gestión más activa de captación de clientes, promoción y ventas que a la vez redundaría en el incremento de los beneficios tangibles y de los indicadores que los soportan (mayor número de participantes, mayor estancia media), los cuales se vinculan desde la perspectiva de los participantes la atracción del evento y del destino y la intención de repitencia como turista convencional o eventista.

\section{Referencias bibliográficas}

Bertoni, M. (2008). Turismo sostenible: su interpretación y alcance operativo. Universidad Nacional de Mar del Plata, Argentina. Cuadernos de Geografía. Revista Colombiana de Geografía. No. 17. Bogotá Colombia. Páginas 155 / 163. Disponible en http:www.scienciedirect.com.

Bueno Campos, E (1998). El Capital Intangible como clave estratégica en la competencia actual. Boletin de estudios económicos. Vol LIII, N 164, pp 207-229.

Drucker, P.F. (1993) La sociedad pos capitalista. Argentina. Editorial Sudamérica SA.

Gandara J. (2001). El turismo y la sostenibilidad de los destinos urbanos

Getz, D. (2008). Progress in TourismManagement 29 (2008) 403-428..Eventtourism: Definition, evolution, and research

Garcia, M., Simo, P., \& Joan Mundet, J. G. (2004). Intangibles: Activos y Pasivos. Management\&Empresa, 32-42. 
Malgor, I (2013). Revista digital La nueva España, segmento para emprendedores, articulo "Recursos intangibles y desarrollo sostenible"

Martínez, M. (2003). El Capital intelectual en un departamento universitario. Análisis el área socio-jurídico. Sevilla, España: Universidad de Sevilla.

Pujol, L., \& Maroto, V. (2003). NTP 640: Indicadores para la valoración de intangibles en prevención. Ministerio de Trabajo y Asuntos Sociales, España.

Revista Special MICE and business 2020 ; "El impacto del Turismo de Reuniones supera los 6.638 millones de euros"

Villa, D. (2014). Capital Relacional y Construcción de ventajas competitivas . Universidad Nacional de Colombia. Facultad de Administración, Manizales, Colombia.

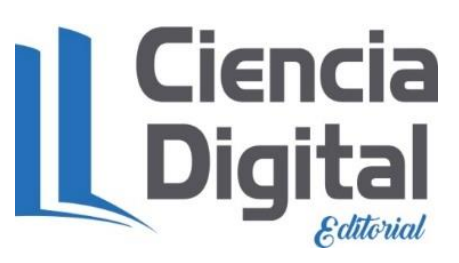




\section{PARA CITAR EL ARTÍCULO INDEXADO.}

Sánchez Fernández, A. (2021). La incidencia de los eventos científico técnicos en los activos intangibles de sus actores. Caso de estudio: pedagogía 2019 : The incidence of technical scientific events on the intangible assets of its actors. Case study: pedagogy 2019. Explorador Digital, 5(1), 470-483. https://doi.org/10.33262/exploradordigital.v5i1.1514

\section{Ciencia \\ LDigital}

El artículo que se publica es de exclusiva responsabilidad de los autores y no necesariamente reflejan el pensamiento de la Revista Explorador Digital.

El artículo queda en propiedad de la revista y, por tanto, su publicación parcial y/o total en otro medio tiene que ser autorizado por el director de la Revista Explorador Digital.
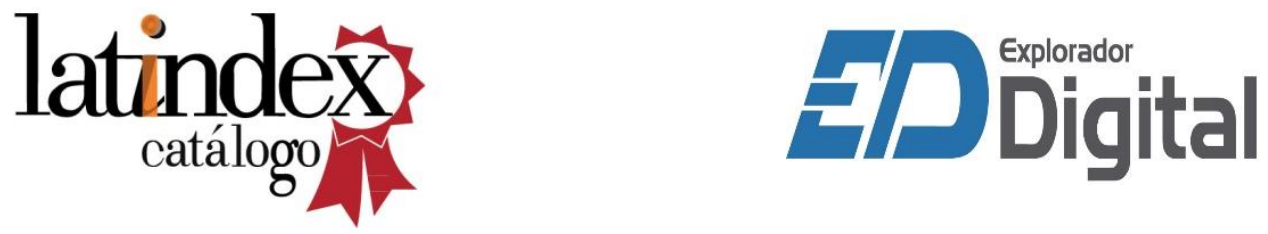